\title{
Frataxin participates to the hypoxia-induced response in tumors
}

\author{
I Guccini ${ }^{1}$, D Serio ${ }^{1}$, I Condò ${ }^{1}$, A Rufini ${ }^{1}$, B Tomassini ${ }^{1}$, A Mangiola ${ }^{2}$, G Maira ${ }^{2}$, C Anile ${ }^{2}$, D Fina ${ }^{3}$, F Pallone ${ }^{3}$, MP Mongiardi ${ }^{4}$, A Levi ${ }^{4}$, \\ N Ventura ${ }^{*, 1}, \mathrm{R}$ Testi $^{*, 1}$ and F Malisan ${ }^{*, 1}$
}

Defective expression of frataxin is responsible for the degenerative disease Friedreich's ataxia. Frataxin is a protein required for cell survival since complete knockout is lethal. Frataxin protects tumor cells against oxidative stress and apoptosis but also acts as a tumor suppressor. The molecular bases of this apparent paradox are missing. We therefore sought to investigate the pathways through which frataxin enhances stress resistance in tumor cells. We found that frataxin expression is upregulated in several tumor cell lines in response to hypoxic stress, a condition often associated with tumor progression. Moreover, frataxin upregulation in response to hypoxia is dependent on hypoxia-inducible factors expression and modulates the activation of the tumor-suppressor p53. Importantly, we show for the first time that frataxin is in fact increased in human tumors in vivo. These results show that frataxin participates to the hypoxia-induced stress response in tumors, thus implying that modulation of its expression could have a critical role in tumor cell survival and/or progression.

Cell Death and Disease (2011) 2, e123; doi:10.1038/cddis.2011.5; published online 24 February 2011

Subject Category: Cancer

Friedreich's ataxia (FRDA) is a degenerative inherited disease because of deficient expression of the nuclearencoded mitochondrial protein frataxin. ${ }^{1}$ Frataxin is required for organismal development, since its complete loss results in embryonic lethality in mouse, ${ }^{2}$ in plant Arabidopsis $^{3}$ and in developmental arrest in the nematode $C$. elegans. ${ }^{4}$ Partial expression of frataxin allows instead organismal development and survival, yet results in progressive degeneration of specific tissues. In fact, frataxin deficiency in humans critically affects survival of large primary neurons of the dorsal root ganglia, cardiomyocytes and pancreatic $\beta$-cells, accounting for FRDA syndromic features. ${ }^{1}$

Frataxin is therefore crucial for cell survival. Although the exact function of this protein is still unclear, it has been implicated in different biological functions, such as iron homeostasis, ${ }^{5}$ iron-sulfur cluster (ISC) biosynthesis, ${ }^{6}$ protection from oxidative stress and apoptosis. ${ }^{7}$ Conflicting results have been reported on the role of frataxin in cellular growth: both frataxin knockdown and frataxin overexpression were shown to impair cell growth. ${ }^{8-11}$ Very little is known regarding the molecular regulation of frataxin expression: histone deacetylase inhibitors, erythropoietin, cisplatin, 3-nitropropionic acid and hemin increase levels of frataxin in vitro (for review, see ref. 12). Moreover, transcription factors peroxisome proliferator-activated receptor gamma, ${ }^{13}$ and hypoxia-inducible factor-2 alpha (HIF-2 $\alpha),{ }^{14}$ positively regulate frataxin expression. However, the molecular bases underlining the potential role of frataxin in cancer are unknown.
Hypoxia is a characteristic feature of most solid tumors that promotes the angiogenic switch necessary for tumor progression. ${ }^{15}$ The hypoxic environment found in tumors is not only dramatically associated with tumor progression but also confers tumor resistance to chemotherapy and radiotherapy. Cells under hypoxic stress can either develop an adaptive response, increasing the rate of glycolysis and angiogenesis, or undergo cell death through apoptosis and/or necrosis. ${ }^{16,17}$ The ability of tumor cells to maintain a balance between adaptation to hypoxia and cell death is mainly mediated by HIFs, which are essential for the regulation of the expression of a large number of hypoxia-responsive genes. ${ }^{16,17} \mathrm{HIFs}$ are heterodimers consisting of either HIF- $1 \alpha$ or HIF- $2 \alpha$ bound to HIF $\beta /$ ARN subunit. Although HIF- $1 \beta$ is constitutively expressed, both $\mathrm{HIF} \alpha$ subunits are modulated by oxygen availability. Under normoxia, HIF $\alpha$ subunits are degraded by the proteasome through binding to the Von Hippel-Lindau E3 ligase. Low oxygen tension stabilizes the $\mathrm{HIF} \alpha$ subunits by inhibiting its degradation thus activating hypoxic adaptation responses. HIFs regulate the expression of genes containing conserved hypoxia-responsive element (HRE). ${ }^{18}$ Murine frataxin was shown to possess an HRE and its expression is indeed controlled by HIF-2 $\alpha .{ }^{14}$ In addition, a direct correlation between upregulation of HIF expression and tumor progression is well documented. ${ }^{16}$

Stabilization of the tumor-suppressor p53 is one of the many cellular responses triggered by hypoxic conditions. ${ }^{19,20}$ p53 itself is indeed modulated by HIF transcription factors ${ }^{21}$ and is involved in the metabolic alterations, which occur in

\footnotetext{
${ }^{1}$ Department of Experimental Medicine and Biochemical Sciences, Laboratory of Signal Transduction, University 'Tor Vergata', Rome, Italy; ${ }^{2}$ Department of Neurosurgery, Catholic University School of Medicine, Rome, Italy; ${ }^{3}$ Department of Internal Medicine, University 'Tor Vergata', Rome, Italy and ${ }^{4}$ National Research Council of Italy, Cell Biology and Neurobiology Institute and IRCCS Fondazione Santa Lucia, Rome, Italy

${ }^{*}$ Corresponding authors: N Ventura or R Testi or F Malisan, Department of Experimental Medicine and Biochemical Sciences, Laboratory of Signal Transduction, University of Rome 'Tor Vergata', Via Montpellier 1, 00133 Rome, Italy. Tel: + 39067259 6501; Fax: + 39067259 6505; E-mail: Natascia.Ventura@med.uniroma2.it (N Ventura) or Roberto.Testi@ uniroma2.it (R Testi) or Malisan@med.uniroma2.it (F Malisan)

Keywords: frataxin; hypoxia; p53; HIF; Friedreich's ataxia; tumor

Abbreviations: FRDA, Friedreich's ataxia; HIF, hypoxia-inducible factor; HRE, hypoxia-responsive element; ISC, iron-sulfur cluster; ROS, reactive oxygen species Received 27.8.10; revised 13.1.11; accepted 19.1.11; Edited by P Salomoni
} 
cancer cells. ${ }^{22}$ Hypoxia associated with tumor progression, not only results in enhanced stabilization of the master regulator HIF- $1 \alpha$ but also in an increased leakage of reactive oxygen species (ROS) from the mitochondrial electron transport chain. ${ }^{23}$ Mitochondrial ROS production in turn activates p53 (ref. 24) while frataxin dampens oxidative stress. ${ }^{25}$ Moreover, a link between frataxin, oxidative stress and p53 has already been observed in $C$. elegans where the absence of $c e p-1$, the $C$. elegans p53 homologue, significantly suppresses the increase in lifespan induced by reduced expression of the frataxin homologue frh-1 (ref. 26). cep-1 also regulates the induction of gst-4 (ref. 27), an antioxidant gene controlled by the redox-transcription factor skn-1, the C. elegans homolog of Nfr2, whose activation is impaired in human cells with defective frataxin expression. ${ }^{28}$

Considering that hypoxia is a major stress signal for tumor cells, and that the murine frataxin is regulated by HIF, we hypothesized that frataxin could participate in tumor adaptation to hypoxia by regulating p53-dependent metabolic pathways. In this study, we found that hypoxia induces frataxin expression in different human tumor cell lines in a HIF-1 $\alpha$ dependent manner. In addition, frataxin silencing in tumor cells prevented hypoxia-induced p53 activation. Our data thus suggest that frataxin expression could have a critical role in tumor cell survival and/or progression. Importantly, in support of this idea, we now provide evidence for an in vivo increase in frataxin in human glioblastoma and colon cancer samples.

\section{Results}

Hypoxia induces frataxin expression in tumor cells. As tumor progression is associated with hypoxia, we analyzed frataxin expression on hypoxic stress in several tumor cell lines, such as two human glioblastoma cell lines U87 and U118, colon carcinoma HCT116 and human epithelial cervical carcinoma HeLa cells. The effect of hypoxia was also tested on immortalized $B$ lymphoblasts derived from a FRDA patient and on control-matched immortalized B lymphoblasts derived from a healthy brother. Cells were subjected to hypoxia $\left(<1 \% \mathrm{O}_{2}\right)$ and collected at the time indicated. Longer exposures to hypoxia resulted in cell death (data not shown). As expected, HIF-1 $\alpha$ protein expression increased on hypoxia compared with untreated cells $\left(\begin{array}{ll}21 \% & \mathrm{O}_{2}\end{array}\right)$ (Figure 1). Importantly, both frataxin protein (Figure 1) and mRNA (Supplementary Figure 1) were significantly upregulated following severe hypoxic stress in all the cell types analyzed. Frataxin expression was induced in the different cell lines at different time points and with different intensity, probably reflecting differential cell-type sensitivity to low oxygen. Of note, transcriptional upregulation of frataxin appears to be modest compared with the effect on protein level. This observation suggests that, consistent with our recent report describing frataxin stability under proteasome control in normoxia, ${ }^{29}$ additional mechanisms for frataxin protein stabilization could also exist in hypoxic conditions.

Hypoxia-induced frataxin upregulation is mediated by HIF. HIFs are main mediators of hypoxia. Under low oxygen conditions, they translocate to the nucleus where they act as

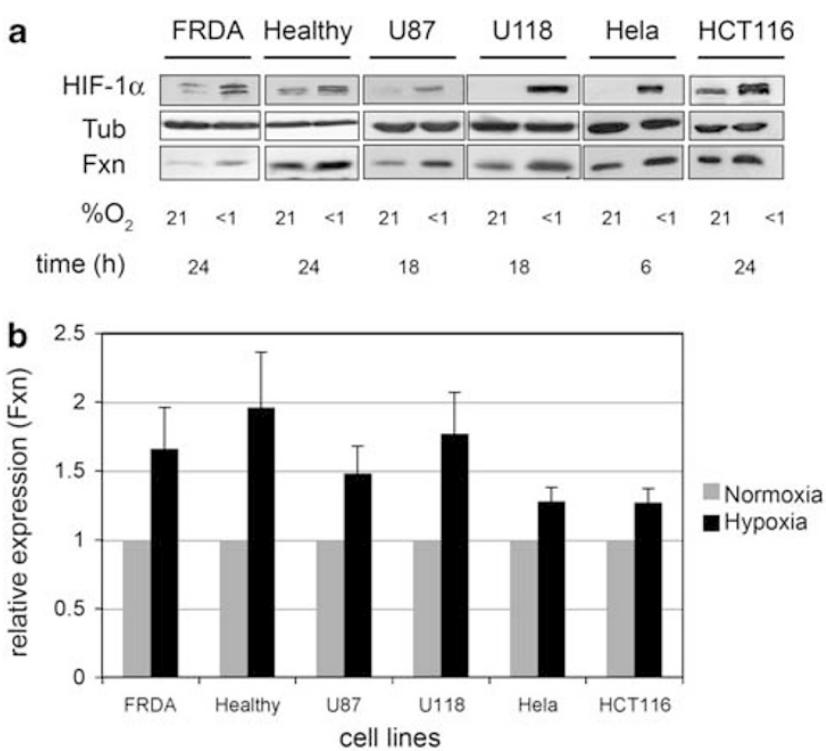

Figure 1 Hypoxic stress upregulates frataxin. (a) FRDA patient-derived $B$ cells (FRDA) and respective control cells (Healthy), human glioblastoma U87, U118, colon carcinoma HCT116 and HeLa cells were placed for different time exposure ( $24 \mathrm{~h}$ for FRDA, healthy control-derived B cells and HCT116, $18 \mathrm{~h}$ for U87, U118 and $6 \mathrm{~h}$ for HeLa) into hypoxic chamber and frataxin (Fxn), tubulin (Tub) and HIF-1 $\alpha$ expression was analyzed by western blot. Data are representative of $6,11,4,7,5$ and 3 independent experiments for FRDA, Healthy, U87, U118, HeLa and HCT116 respectively. (b) Densitometric quantification of frataxin upregulation. Frataxin expression was normalized with tubulin and frataxin expression in normoxia set to one. Data represent the mean \pm 1 S.E.M. from the different independent experiments performed for each cell line described in a. $P$-values were calculated with Student's $t$-test and were statistically significant $(P<0.05)$ for all cell lines analyzed

transcription factors for different HRE-containing genes. As murine frataxin was shown to possess an HRE for HIF- $2 \alpha$, we assessed whether frataxin upregulation in response to hypoxia in human cancer cells is mediated through the HIF pathway. We took advantage of glioblastoma cell lines TB10, U87 and U118, which stably express shRNA against HIF-1 $\alpha$, resulting in $70-80 \%$ knockdown of its expression. ${ }^{30}$ Wildtype cells and cells stably expressing shHIF-1 $\alpha$ were subjected to hypoxia and frataxin expression was analyzed. As expected, on shRNA treatment, hypoxia-induced HIF-1 $\alpha$ expression was significantly prevented in cells stably expressing shHIF-1 $\alpha$. Importantly, while HIF-2 $\alpha$ expression was unchanged, the induction of frataxin on hypoxic stress was almost completely abolished (Figure 2). These results suggest that frataxin induction in response to hypoxia is controlled by HIF-1 $\alpha$ in human cells.

Frataxin deficiency differently affects p53 activation in normoxia or hypoxia. Hypoxia modulates the activation of the tumor-suppressor p53, one of the most important defender against tumor development. ${ }^{22}$ We analyzed hypoxia-induced p53 activation in immortalized B lymphoblasts derived from a FRDA patient, compared with controlmatched immortalized B lymphoblasts derived from a healthy brother. Cells undergoing hypoxia showed p53 phosphorylation at ser15, a classical marker of p53 activation. Interestingly, frataxin-defective cells had increased basal 
a

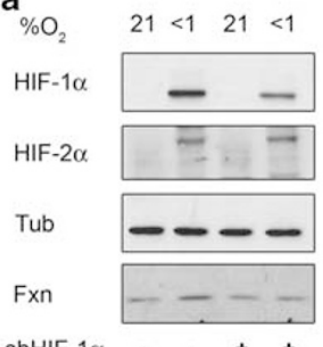

shHIF-1 $\alpha-\cdot++$

b

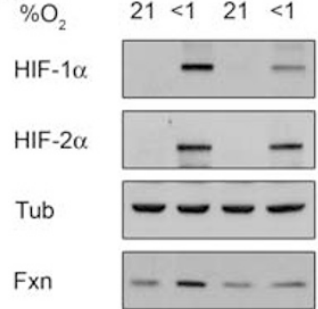

ShHIF-1 $\alpha+-++$

C

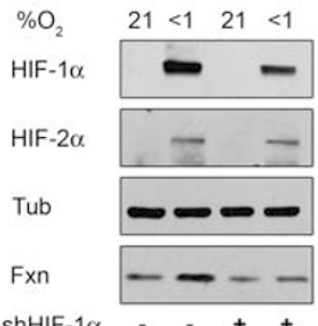

shHIF- $1 \alpha$

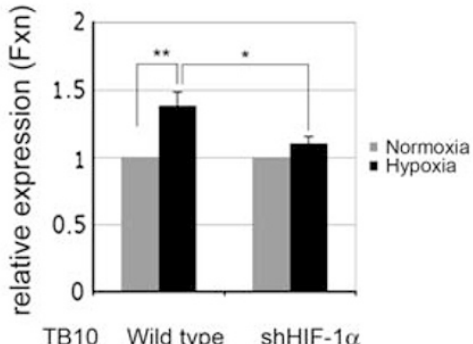

TB10 Wild type shHIF-1 $\alpha$

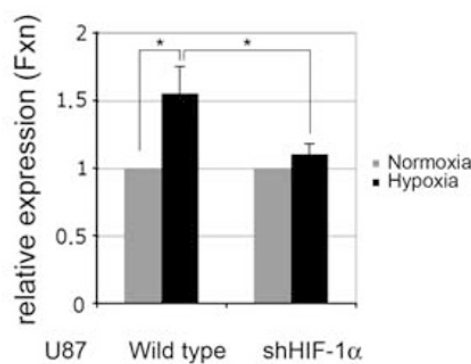

Figure 2 HIFs mediate hypoxia-induced frataxin upregulation. Left panels: human glioblastoma cells TB10 (a), U87 (b) and U118 (c) wild type or stably interfered for HIF-1 $\alpha$ (shHIF-1 $\alpha$ ) were exposed to severe hypoxia for $18 \mathrm{~h}$ and frataxin (Fxn), tubulin (Tub), HIF-1 $\alpha$ and HIF-2 $\alpha$ expression analyzed. Data are representative of four, three and three independent experiments for TB10, U87 and U118 cells, respectively. Right panels: densitometric quantification of frataxin upregulation. Frataxin expression was normalized with tubulin and frataxin expression in normoxia set to one. Data represent the mean \pm 1 S.E.M. from the different independent experiments performed for cell lines described in left panels. $P$-values were calculated with Student's $t$-test: ${ }^{*} P<0.05$; ${ }^{* \star} P<0.01$

level of phosphorylated p53 but a less efficient activation of p53 on hypoxia (Figure 3a).

To further address the role of frataxin in modulating p53, we also interfered with frataxin expression using a shRNA-based approach in HeLa and U118 cell line, achieving a 60-70\% frataxin knockdown. Cells were then placed into hypoxic conditions and p53 activation was analyzed by western blot. Similarly to FRDA cells, frataxin knockdown HeLa (Figure 3b) and U118 (Figure 3c) cells had increased basal level of phosphorylated p53 compared with control cells. On hypoxia exposure, however, both HeLa (Figure $3 b$ ) and U118 (Figure 3c) failed to upregulate frataxin and to activate p53. Thus, cells with slightly activated p53 might respond less efficiently to hypoxia. Importantly, normal frataxin levels appear to be required for optimal p53 activation following hypoxia.

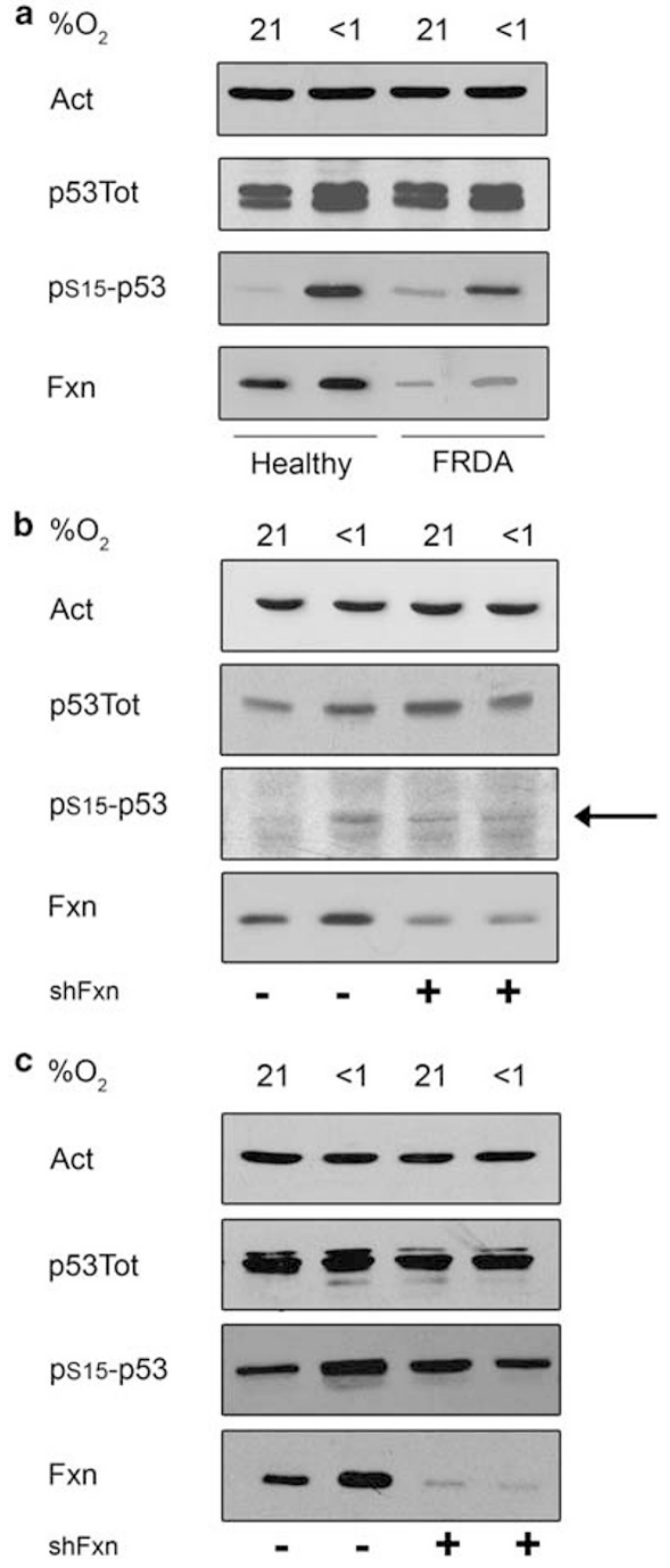

Figure 3 Frataxin deficiency prevents p53 activation in response to hypoxia. (a) FRDA patient-derived $\mathrm{B}$ cells (FRDA) and respective control cells (Healthy) were placed into hypoxic chamber for $18 \mathrm{~h}$ and frataxin (Fxn), actin (Act), total p53 (p53Tot) and phosphorylation at serine 15 of p53 (pS15-p53) expression analyzed. Data are representative of six independent experiments. (b) HeLa cells were transiently transfected with plasmid for frataxin gene interference (shFxn) each 2 days. Frataxin (Fxn), actin (Act), total p53 (p53Tot) and phosphorylation at serine 15 of p53 (pS15-p53) were analyzed after three rounds of transfection and exposure or not to hypoxic conditions for $18 \mathrm{~h}$. Data are representative of three independent experiments (c) hFxnRNAi and control U118 cells were treated for 5 days with doxycycline. Frataxin (Fxn), actin (Act), total p53 (p53) and phosphorylation at serine 15 of p53 (pS15-p53) expression were analyzed after exposure or not to hypoxia for $18 \mathrm{~h}$. Data are representative of four independent experiments

Frataxin is upregulated in human tumor samples in vivo. To assess whether frataxin levels are modulated in hypoxic tissues in vivo, frataxin was quantitated in human glioblastoma and colon carcinoma tumor samples. Analysis was performed comparing central tumor sample with the 

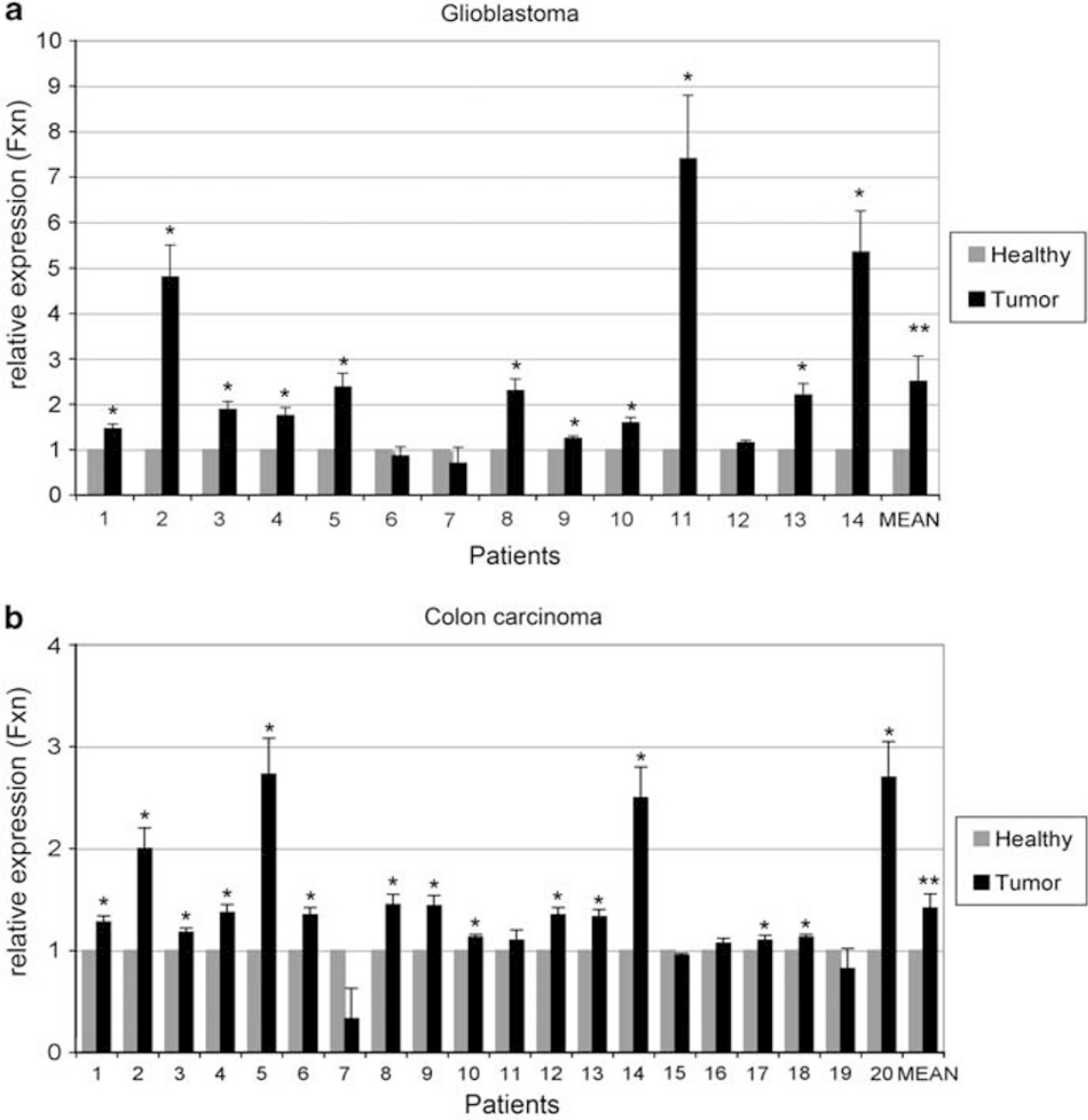

Figure 4 Frataxin is upregulated in human glioblastoma and colon cancer. Protein extracts $(100 \mu \mathrm{g})$ were prepared from human glioblastoma and colorectal carcinoma (Tumor) and corresponding normal samples (Healthy). Frataxin expression was analyzed by western blot and normalized with tubulin and $\beta$-actin for glioblastoma (a) and colon carcinoma (b), respectively, and frataxin expression in healthy samples set to one. The densitometric quantification of normalized frataxin in the various samples as well as mean upregulation is indicated (mean \pm 1 S.E.M.). Statistical analysis was performed by Student's $t$-test: ${ }^{*} P<0.05 ;{ }^{*} P<0.01$

adjacent healthy tissue of the same patient. Strikingly, frataxin resulted to be significantly upregulated in $>85 \%$ of tumor samples analyzed (Figures $4 a$ and b). Frataxin expression was upregulated in 12 out of 14 glioblastoma (Figure $4 \mathrm{a}$ ), and in 17 out of 20 colorectal carcinoma tumor samples (Figure 4b), compared with the adjacent healthy tissue. The average frataxin upregulation resulted in 2.50- and 1.42-fold increase in glioblastoma and colorectal carcinoma tumors, respectively, and was statistically significant both in glioblastomas and colorectal carcinomas $(P<0.01)$.

In one case of glioblastoma, we first obtained surgical resections from the primary tumor and then from a relapse. In the relapse samples, the tumor core presented extended necrotic areas, a feature usually associated to severely hypoxic tissue and the adjacent peripheral tissue was infiltrated by neoplastic cells. Interestingly, frataxin expression was higher in the relapse sample, compared with the primary sample, both in the core and in the adjacent peripheral areas (Figure 5a).

In addition, for other six glioblastoma patients, two peripheral healthy samples adjacent to the tumor resection edge were collected. P1 is closer than $\mathrm{P}$ to the tumor central core. Interestingly, we could correlate frataxin expression level with the distance to the tumor core sample: the closer is the peripheral sample to the tumor core, the higher is the frataxin expression (Figure $5 b$ ).

Moreover, although the lack of information regarding p53 mutations in the samples analyzed does not allow to directly link frataxin expression to p53 activation, according to what we observed in human cell lines undergoing hypoxia, frataxin upregulation correlated with p53 expression and activation (Supplementary Figure 2).

\section{Discussion}

This study shows that frataxin is upregulated on hypoxia in tumor cell lines and that this event is dependent on HIF-1 $\alpha$. Moreover, frataxin is required for optimal p53 activation following hypoxia in tumor cell lines. Importantly, here we report that frataxin is in fact upregulated in vivo in human glioblastoma and colon tumor samples.

Tumor proliferation depends on adequate nutrients and oxygen supply but tumor cells also need to survive under hostile conditions that include hypoxia and increased oxidative stress. The ability of tumor cells to maintain a balance 
a
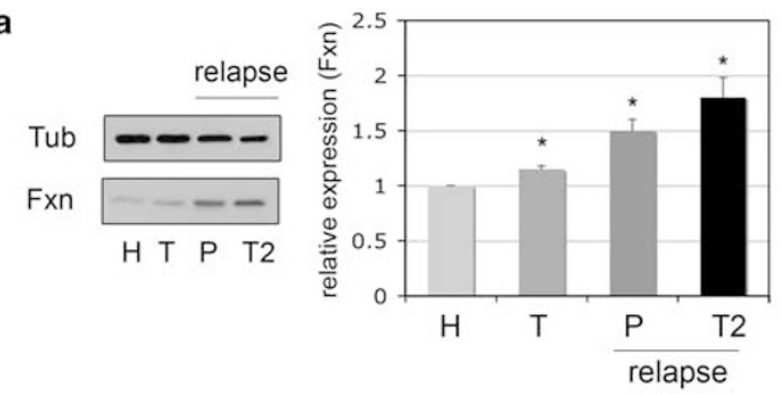

b

\section{Tub
Fxn}
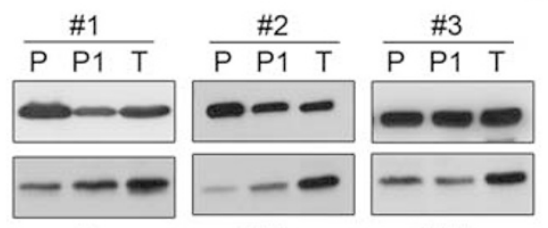

$\# 13$
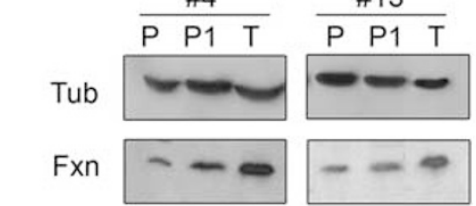

\#14

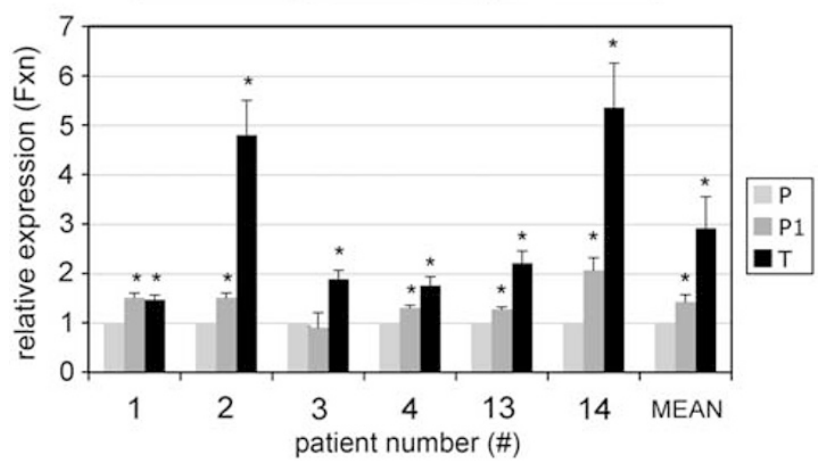

Figure 5 A correlation between frataxin expression and tumor progression could be found in different human glioblastoma patients. Protein extracts $(100 \mu \mathrm{g})$ were prepared from human glioblastoma and corresponding normal samples. Frataxin (Fxn) expression was normalized with tubulin (Tub) and frataxin expression in healthy samples set to one. (a) Frataxin expression was analyzed in patient healthy $(H)$ and tumoral $(T)$ samples after first surgical resection and in neoplastic infiltrated peripheral tissue $(\mathrm{P})$ and tumoral (T2) samples after second surgical resection because of tumor relapse. (b) Frataxin was analyzed in six different peripheral ( $P$ and $P 1)$ samples. $P 1$ is closer to central tumor core $(T)$. Patient numbers $(1,2,3,4,13$ and 14) are referred to Figure 4a. The densitometric quantification of normalized frataxin in the various samples as well as mean upregulation is indicated (mean \pm 1 S.E.M.). Statistical analysis was performed by Student's t-test: ${ }^{*} P<0.05$

between adaptation to hypoxia and cell death is mainly mediated by transcription factors HIFs. Moreover, mitochondrial alterations including frataxin downregulation are observed in HIF- $2 \alpha$ knockout mice, ${ }^{31}$ and murine frataxin promoter is under control of HIF-2 $\alpha .{ }^{14}$ We report that frataxin levels are increased in vivo both in human colon carcinoma and human glioblastoma tumors. Consistent with the HIF upregulation observed in tumors, especially in human glioma tissues, ${ }^{16,32}$ we found that hypoxia induces frataxin accumulation in a HIF-1 $\alpha$-dependent manner in human glioblastoma TB10, U87 and U118 cell lines.
Frataxin binds iron and is involved in iron metabolism. ${ }^{33}$ Considering that iron controls HIFs degradation and that HIFs, in turn, can regulate iron metabolism modulating the levels of hepcidin, a functional interplay between HIFs and frataxin is expected. Although frataxin deficiency was reported to result in upregulation of HIF- $1 \alpha$ proteins in cardiomyocytes of conditional frataxin knockout mutant mice with evident cardiomyopathy, ${ }^{34}$ we could not detect any significant differences of HIFs protein expression between healthy control and FRDA lymphoblasts or between wild-type and shFxn HeLa and U118 cells, both in normoxia and hypoxia conditions. Moreover, a role for HIF- $2 \alpha$ but not HIF- $1 \alpha$ in iron absorption of intestinal epithelium in mice has been reported: only HIF- $2 \alpha$ can regulate the iron transporter DMT1 (ref. 35). It is noteworthy that contrasting effects between HIF- $1 \alpha$ and HIF- $2 \alpha$ have also been described on 053 activation in human cells. Although HIF- $1 \alpha$ activates p53 (ref. 19), HIF- $2 \alpha$ has been recently reported to inhibit p53 and ROS production. ${ }^{21}$ Considering that murine frataxin is controlled by HIF- $2 \alpha$ but that in our cell system human frataxin upregulation in response to hypoxia depends primarily on HIF- $1 \alpha$, it would be interesting to further investigate whether such a discrepancy depends on cell and/or tissue type or on species differences.

Given the role of frataxin in ISC biogenesis, the HIF- $1 \alpha$ mediated frataxin upregulation is apparently in contrast with the pathways leading to mitochondrial dysfunctions in hypoxic cells. ${ }^{17}$ In this context, miR-210, which is a key microRNA upregulated by HIF-1 $\alpha$ under hypoxic conditions, leads to downregulation of the scaffold protein ISCU in order to repress the mitochondrial electron transport and the Krebs' cycle. $^{36}$ However, the repression of ISCU by miR-210 concomitantly contributes to the generation of ROS during hypoxia, ${ }^{36,37}$ and frataxin, in addition to its function as iron chaperone, also has a key function in the adaptive response to oxidative stress. ${ }^{25}$ As oxidative stress correlates with aggressiveness of tumors and promotes tumor cell survival under hypoxic conditions, ${ }^{15}$ frataxin induction may contribute to tumor cells survival under hypoxia by regulating cells antioxidant response. A fine-tuned regulation already exists between hypoxia, HIF, p53 activation and ROS production: hypoxia stimulates mitochondrial ROS production, which modulates HIF- $1 \alpha^{38}$ and HIF- $1 \alpha$, in turn, has a crucial homeostatic role in managing oxygen consumption to balance ATP and ROS production. ${ }^{17}$ Moreover, hypoxia activates p53, which has both pro-oxidant and anti-oxidant effects. ${ }^{22}$ We now add a new player to this complex loop by providing data showing that frataxin deficiency induces p53 activation in normoxia while preventing it on hypoxia. Frataxin deficiency in FRDA patients leads to increased ROS production, ${ }^{25}$ which could in turn activate p53 resembling hypoxic stress condition. This basal stress could then interfere with further adequate adaptive hypoxic responses preventing optimal p53 activation. In support of our findings, several evidences point to differential p53 biological responses depending on the degree of damage or stress as a conserved evolutionary mechanism from nematodes to mammals. ${ }^{22,26}$ Moreover, consistent with a role of frataxin in regulating p53 responses, we have previously shown that frataxin deficiency modulates longevity and antioxidant gst-4 activation in C. elegans, 
in a p53-dependent manner ${ }^{26,27}$ and a recent report describes partners proteins of p53 and ATM significantly altered in FRDA patients. ${ }^{39}$ Overall, frataxin levels may contribute to the survival pathways of tumor cells via a fine-tuned regulation of the anti-oxidant response under hypoxic environment, likely influencing metabolic pathways by modulating the hypoxiainduced p53 stress response. The mechanisms underlying differential modulation of $p 53$ activation by frataxin expression levels in normoxia and hypoxia with possible consequent recruitment of different p53 downstream targets will require further investigation.

Last but not least, considering that in FRDA, frataxin expression is inversely correlated with disease onset, progression and severity, it is noteworthy that frataxin protein level could be upregulated on hypoxia even in FRDA patient derived lymphoblastoid cells. Although hypoxia cannot be considered as a therapeutic strategy for this disease, further unraveling the mechanisms contributing to hypoxia-induced frataxin upregulation could be crucial to identify novel drugs capable of increasing frataxin level in FRDA patients.

In summary, frataxin is crucial for cell life since reduction of its expression leads to neurodegenerative disease FRDA and complete knockout is lethal in several organisms such as mice and plants. Frataxin overexpression can protect tumor cells from apoptosis but can also act as a tumor suppressor. We herein provide explanation for this apparent paradox showing that frataxin participates, by regulating p53 activation, in tumor adaptation to hypoxia, a critical feature associated with tumor growth and progression, thus suggesting that frataxin levels can influence tumor cell fate. We show indeed for the first time that frataxin is increased in vivo in human tumor tissues.

\section{Materials and Methods \\ shRNA construct (shFxn). Frataxin was depleted in human cells by RNAi using a pSUPER vector-based approach. Self-complementary inverted repeat sequences of frataxin (19 nucleotide long frataxin gene-specific targeting sequence $5^{\prime}$ GTTCGAACCAACGTGGCCT-3', position 167-185 of the coding sequence), spaced by 9 base loop region $5^{\prime}$ TTCAAGAGA-3' were synthesized (Invitrogen, San Giuliano Milanese, Italy) as single-strand oligonucleotides. These 64 base long oligonucleotides containing the coding and complementary sequence of these components, together with $\mathrm{Bg} / \mathrm{II}$ and Hindlll restriction site sequences at the $5^{\prime}$ and $3^{\prime}$ ends respectively, were then annealed by heating to $89^{\circ} \mathrm{C} 10 \mathrm{~min}$ and cooled down to $69^{\circ} \mathrm{C}$ in $30 \mathrm{~min}$, and then to $65^{\circ} \mathrm{C} 10 \mathrm{~min}$, in $50 \mathrm{mM}$ Tris- $\mathrm{HCl}, 10 \mathrm{mM}$ $\mathrm{MgCl}_{2}, 10 \mathrm{mM}$ DTT, $1 \mathrm{mM}$ ATP, $25 \mathrm{mg} / \mathrm{ml} \mathrm{BSA}, 10 \%$ DMSO. Successful annealing was checked by agarose gel electrophoresis, and the obtained double-stranded DNA was cloned into the pSUPER vector via the Bg/II and HindllI restriction sites. All constructs were checked by DNA sequencing.}

The inducible $\mathrm{hFxnRNAi}$ construct (pSUPERIOR.PURO vector) and pcDNA6/TR plasmid were a kind gift of Dr. Cortopassi. ${ }^{40}$

Cell culture, transfections and hypoxia. Immortalized GM15850B lymphoblasts, from a clinically affected FRDA patient, and immortalized GM15851B lymphoblasts from a clinically unaffected brother of GM15850 were obtained from NIGMS Human Genetic Cell Repository, Coriell Institute for Medical Research (Camden, NJ, USA) and were cultured in RPMI 1640 supplemented with $15 \%$ fetal bovine serum. HeLa, U87, U118, HCT116 and TB10 cells were cultured in DMEM supplemented with $10 \%$ fetal bovine serum.

When described, these cells were transiently transfected with DNA constructs using Lipofectamine 2000 (Invitrogen), following the manufacturer's instructions. To interfere with frataxin expression, HeLa cells were transfected with shRNA plasmid for frataxin gene (shFxn). Three rounds of transfection every 2 days were performed to achieve a $60-70 \%$ frataxin knockdown.
To create severe hypoxic environmental conditions, cells were placed into a modular incubator chamber (Billups-Rothenberg, Del Mar, CA, USA) and gas without oxygen $\left(95 \% \mathrm{~N}_{2}, 5 \% \mathrm{CO}_{2}\right)$ was flushed into the chamber for $10 \mathrm{~min}$. Cells were then cultured into a normal $\mathrm{CO}_{2}$ incubator for different time periods.

Generation of stably shHIF- $1 \alpha$ cell lines. Glioblastoma cell lines TB10, U87 and U118 with silencing of HIF- $1 \alpha$ were obtained, as previously described, by retroviral mediated expression of shRNA targeting nucleotide specific for HIF-1 $\alpha$ sequence resulting in $70-80 \%$ knockdown of its expression. ${ }^{30}$

Generation of stable doxycycline/tetracycline-inducible hFxnRNAi cell line. Human U118 glioblastoma cells stably transfected using Lipofectamine 2000 (Invitrogen) with pcDNA6/TR plasmid, therefore expressing the tetracycline repressor, were first generated by 10 days selection with blasticidin hydrochloride (Invitrogen) $2 \mu \mathrm{g} / \mathrm{ml}$. Cells were maintained in DMEM supplemented with $10 \%$ tetracycline-free fetal bovine serum (HyClone, Rockford, IL, USA).

Stably expressing pcDNA6/TR U118 cells were then transfected with inducible hFxnRNAi construct or the empty pSUPERIOR.PURO vector as a control for the inducible system. At $24 \mathrm{~h}$ after transfection, the cells were split and grown in medium containing $1.5 \mu \mathrm{g} / \mathrm{ml}$ puromycin (Sigma, Milan, Italy). Single clones were isolated and expanded.

The efficiency of the inducible RNAi system was established analyzing frataxin mRNA levels by quantitative real-time PCR after 5 days of treatment with $8 \mu \mathrm{g} / \mathrm{ml}$ doxycycline (Sigma) of hFxnRNAi and control clones. Three hFxnRNAi clones showing $>70-80 \%$ frataxin reduction were selected, as well as control clones in which frataxin level remained unchanged (data not shown).

Quantitative RT-PCR analysis. Total RNA $(500 \mathrm{ng})$ was retrotranscribed using SuperScript VILO cDNA Synthesis Kit (Invitrogen) according to the manufacturer's instructions. One-twentieth of the CDNA was used to quantify the transcripts by real-time RT-PCR employing the 7900HT Fast Real-Time PCR System by Applied Biosystem (Carlsbad, CA, USA). Gene specific-primers were designed by the Probe Finder software (Roche Applied Science, Milan, Italy) and the CDNA was amplified and quantified using the SensiMix dT kit by Quantace (Bioline, London, UK) according to the manufacturer's instructions. The tata-binding protein $\mathrm{mRNA}$ was used as housekeeping invariant reference gene and the relative quantity was calculated accordingly. Specific forward primer for frataxin is situated in the third exon: $5^{\prime}$-CATACACGTTTGAGGACTATGATGTCT-3' and specific reverse primer situated in the fourth exon: $5^{\prime}$-TTTGGCGTCTGCTTGTTGATC- $3^{\prime}$.

Western blot. Total cell extracts were prepared in ice-cold modified RIPA lysis buffer (10 mM sodium phosphate $\mathrm{pH} 7.2,150 \mathrm{mM} \mathrm{NaCl}, 1 \% \mathrm{NP}-40,1 \%$ sodium deoxycholate, $0.1 \%$ SDS, 2 mM EDTA) supplemented with complete protease inhibitor cocktail (Roche Diagnostics, Milan, Italy). Cell lysates $(100 \mu \mathrm{g})$ were resolved by SDSPAGE and analyzed by immunoblot with specific mAb anti-frataxin (Immunological Sciences, Rome, Italy), mAb anti-tubulin (Sigma), mAb anti- HIF-1 $\alpha$ (Becton Dickinson, Milan, Italy), mAb anti- HIF-2 $\alpha$ (Santa Cruz Biotechnology, Heidelberg, Germany), mAb anti- $\beta$ actin (Sigma), mAb anti p53 (Santa Cruz Biotechnology) mAb anti phosphorylated p53 (ser 15) (Cell Signaling Technology, Danvers, MA, USA) using ECL system detection (GE Healthcare Europe $\mathrm{GmbH}$, Milan, Italy). Densitometric analyses were performed using ImageJ software (W Rasband, National Institutes of Health, Bethesda, MD, USA; http://rsb.info.nih.gov/ij).

Total cell extracts from human tumor samples. Colorectal adenocarcinoma and corresponding normal tissue from the same patients were obtained from 20 patients who underwent surgical resection. Glioblastoma tissue and corresponding normal counterpart adjacent to the tumor (resection edge) from the same patients were obtained from 14 patients who underwent surgical resection. The normal corresponding tissues were confirmed to be tumor-free by histological analysis. Each sample was frozen in liquid nitrogen, stored at $-80^{\circ} \mathrm{C}$, and subsequently homogenized on ice, in ice-cold modified RIPA lysis buffer supplemented with complete protease inhibitor cocktail. Frataxin, p53 and p53 phosphorylation were analyzed by western blot. Protein expression in human tumor samples was tested in duplicates or triplicates when possible.

Statistical analysis. The significance of differences between populations of data were assessed according to the Student's $t$-test with a level of significance of at least $P<0.05$ (alpha conventionally equal to 0.05 ). 


\section{Conflict of Interest}

The authors declare no conflict of interest.

Acknowledgements. This work was funded in part by FIRB 2003 and Atena Onlus Association for GM, FILAS grant from Regione Lazio for AL, National Ataxia Foundation, Ataxia UK, Friedreich's Ataxia Research Alliance, and Telethon-Italy (Grant GGP06059) for RT. IG is a fellowship holder of the Association Française de l'Ataxie de Friedreich (AFAF).

1. Schmucker S, Puccio H. Understanding the molecular mechanisms of Friedreich's ataxia to develop therapeutic approaches. Hum Mol Genet 2010; 19: R103-R110.

2. Cossee M, Puccio H, Gansmuller A, Koutnikova H, Dierich A, LeMeur M et al. Inactivation of the Friedreich ataxia mouse gene leads to early embryonic lethality without iron accumulation. Hum Mol Genet 2000; 9: 1219-1226.

3. Vazzola V, Losa A, Soave C, Murgia I. Knockout of frataxin gene causes embryo lethality in Arabidopsis. FEBS Lett 2007; 581: 667-672.

4. Ventura N, Rea S, Henderson ST, Condo I, Johnson TE, Testi R. Reduced expression of frataxin extends the lifespan of Caenorhabditis elegans. Aging Cell 2005; 4: 109-112.

5. Richardson DR, Huang ML, Whitnall M, Becker EM, Ponka P, Rahmanto YS. The ins and outs of mitochondrial iron-loading: the metabolic defect in Friedreich's ataxia. $J \mathrm{Mol} M$ 2010; 88: 323-329.

6. Stemmler TL, Lesuisse E, Pain D, Dancis A. Frataxin and mitochondrial Fe-S cluste biogenesis. J Biol Chem 2010; 285: 26737-26743.

7. Condo I, Ventura N, Malisan F, Tomassini B, Testi R. A pool of extramitochondrial frataxin that promotes cell survival. J Biol Chem 2006; 281: 16750-16756.

8. Stehling O, Elsasser HP, Bruckel B, Muhlenhoff U, Lill R. Iron-sulfur protein maturation in human cells: evidence for a function of frataxin. Hum Mol Genet 2004; 13: 3007-3015.

9. Thierbach R, Schulz TJ, Isken F, Voigt A, Mietzner B, Drewes G et al. Targeted disruption of hepatic frataxin expression causes impaired mitochondrial function, decreased life span and tumor growth in mice. Hum Mol Genet 2005; 14: 3857-3864.

10. Calmels N, Seznec H, Villa P, Reutenauer L, Hibert M, Haiech J et al. Limitations in a frataxin knockdown cell model for Friedreich ataxia in a high-throughput drug screen. BMC Neurol 2009; 9: 46

11. Schulz TJ, Thierbach R, Voigt A, Drewes G, Mietzner B, Steinberg $P$ et al. Induction of oxidative metabolism by mitochondrial frataxin inhibits cancer growth: Otto Warburg revisited. J Biol Chem 2006; 281: 977-981.

12. Gottesfeld JM. Small molecules affecting transcription in Friedreich ataxia. Pharmacol Ther 2007; 116: 236-248

13. Marmolino D, Acquaviva F, Pinelli M, Monticelli A, Castaldo I, Filla A et al. PPAR-gamma agonist Azelaoyl PAF increases frataxin protein and mRNA expression: new implications for the Friedreich's ataxia therapy. Cerebellum 2009; 8: 98-103.

14. Oktay Y, Dioum E, Matsuzaki S, Ding K, Yan LJ, Haller RG et al. Hypoxia-inducible facto 2alpha regulates expression of the mitochondrial aconitase chaperone protein frataxin. J Biol Chem 2007; 282: 11750-11756.

15. Michieli P. Hypoxia, angiogenesis and cancer therapy: to breathe or not to breathe? Cell Cycle 2009; 8: 3291-3296.

16. Semenza GL. Defining the role of hypoxia-inducible factor 1 in cancer biology and therapeutics. Oncogene 2010; 29: 625-634.

17. Semenza GL. HIF-1: upstream and downstream of cancer metabolism. Curr Opin Genet Dev 2010; 20: 51-56.

18. Lofstedt T, Fredlund E, Holmquist-Mengelbier L, Pietras A, Ovenberger M, Poellinger L et al. Hypoxia inducible factor-2alpha in cancer. Cell Cycle 2007; 6: 919-926.

19. An WG, Kanekal M, Simon MC, Maltepe E, Blagosklonny MV, Neckers LM. Stabilization of wild-type p53 by hypoxia-inducible factor 1alpha. Nature 1998; 392: 405-408.

20. Schmid T, Zhou J, Brune B. HIF-1 and p53: communication of transcription factors under hypoxia. J Cell Mol Med 2004; 8: 423-431.

21. Bertout JA, Majmundar AJ, Gordan JD, Lam JC, Ditsworth D, Keith B et al. HIF2alpha inhibition promotes p53 pathway activity, tumor cell death, and radiation responses. Proc Natl Acad Sci USA 2009; 106: 14391-14396.

22. Vousden KH, Ryan KM. p53 and metabolism. Nat Rev Cancer 2009; 9: 691-700.

23. Solaini G, Baracca A, Lenaz G, Sgarbi G. Hypoxia and mitochondrial oxidative metabolism. Biochim Biophys Acta 2010; 1797: 1171-1177.

24. Karawajew L, Rhein P, Czerwony G, Ludwig WD. Stress-induced activation of the p53 tumor suppressor in leukemia cells and normal lymphocytes requires mitochondrial activity and reactive oxygen species. Blood 2005; 105: 4767-4775.

25. Armstrong JS, Khdour O, Hecht SM. Does oxidative stress contribute to the pathology of Friedreich's ataxia? A radical question. Faseb J 2010; 24: 2152-2163.

26. Ventura N, Rea SL, Schiavi A, Torgovnick A, Testi R, Johnson TE. p53/CEP-1 increases or decreases lifespan, depending on level of mitochondrial bioenergetic stress. Aging Cell 2009; 8: 380-393.

27. Torgovnick A, Schiavi A, Testi R, Ventura N. A role for $p 53$ in mitochondrial stress response control of longevity in C. elegans. Exp Gerontol 2010; 45: 550-557.

28. Paupe V, Dassa EP, Goncalves S, Auchere F, Lonn M, Holmgren A et al. Impaired nuclea Nif2 translocation undermines the oxidative stress response in Friedreich ataxia. PLOS One 2009; 4: e4253.

29. Rufini A, Fortuni S, Arcuri G, Condo I, Serio D, Incani O et al. Preventing the ubiquitin proteasome-dependent degradation of frataxin, the protein defective in Friedreich's Ataxia. Hum Mol Genet 2011, doi: 10.1093/hmg/ddq566.

30. Fiorenzo P, Mongiardi MP, Dimitri D, Cozzolino M, Ferri A, Montano N et al. HIF1-positive and HIF1-negative glioblastoma cells compete in vitro but cooperate in tumor growth in vivo. Int J Oncol 2010; 36: 785-791.

31. Scortegagna M, Ding K, Oktay Y, Gaur A, Thurmond F, Yan LJ et al. Multiple organ pathology, metabolic abnormalities and impaired homeostasis of reactive oxygen species in Epas1-/- mice. Nat Genet 2003; 35: 331-340.

32. Lu H, Li Y, Shu M, Tang J, Huang Y, Zhou Y et al. Hypoxia-inducible factor-1alpha blocks differentiation of malignant gliomas. FEBS J 2009; 276: 7291-7304.

33. Lane DJ, Richardson DR. Frataxin, a molecule of mystery: trading stability for function in its iron-binding site. Biochem J 2010; 426: e1-e3.

34. Huang ML, Becker EM, Whitnall M, Rahmanto YS, Ponka P, Richardson DR. Elucidation of the mechanism of mitochondrial iron loading in Friedreich's ataxia by analysis of a mouse mutant. Proc Natl Acad Sci USA 2009; 106: 16381-16386.

35. Mastrogiannaki M, Matak P, Keith B, Simon MC, Vaulont S, Peyssonnaux C. HIF-2alpha, but not HIF-1alpha, promotes iron absorption in mice. J Clin Invest 2009; 119: 1159-1166.

36. Chan SY, Zhang YY, Hemann C, Mahoney CE, Zweier JL, Loscalzo J. MicroRNA-210 controls mitochondrial metabolism during hypoxia by repressing the iron-sulfur cluster assembly proteins ISCU1/2. Cell Metab 2009; 10: 273-284.

37. Favaro E, Ramachandran A, McCormick R, Gee H, Blancher C, Crosby M et al. MicroRNA210 regulates mitochondrial free radical response to hypoxia and krebs cycle in cancer cells by targeting iron sulfur cluster protein ISCU. PLOS One 2010; 5: e10345.

38. Taylor CT. Mitochondria and cellular oxygen sensing in the HIF pathway. Biochem $J$ 2008; 409: 19-26.

39. Haugen AC, Di Prospero NA, Parker JS, Fannin RD, Chou J, Meyer JN et al. Altered gene expression and DNA damage in peripheral blood cells from Friedreich's ataxia patients: cellular model of pathology. PLoS Genet 2010; 6: e1000812.

40. Lu C, Cortopassi G. Frataxin knockdown causes loss of cytoplasmic iron-sulfur cluster functions, redox alterations and induction of heme transcripts. Arch Biochem Biophys 2007: 457: 111-122.

(c) licensed under the Creative Commons Attribution-Noncommercial-No Derivative Works 3.0 Unported License. To view a copy of this license, visit http://creativecommons.org/licenses/by-nc-nd/3.0/

Supplementary Information accompanies the paper on Cell Death and Disease website (http://www.nature.com/cddis) 